\title{
Mathematical Interpretation of Open-Pit Dump Trucks Diesel Engine Durability
}

\author{
Kairatbek Yesbossynov ${ }^{1}$, Gennady Buyalich ${ }^{2, *}$, Sapar Kabikenov ${ }^{1}$, Nurbolat Sembayev $^{3}$, \\ and Medet Jailaubekov ${ }^{1}$ \\ ${ }^{1}$ Karaganda State Technical University, Republic of Kazakhstan \\ ${ }^{2}$ T.F. Gorbachev Kuzbass State Technical University, Russia, 65000028 Vesennya st., Kemerovo, \\ Russia \\ ${ }^{3}$ S.Toraighyrov Pavlodar State University, Republic of Kazakhstan
}

\begin{abstract}
The durability of internal combustion engines is determined by the engine operating time, which is established by the service life of the most critical parts and assemblies that undergo normal mechanical abrasion in the process of engine operation. Factory diesel repair is performed when there is a need to repair the crankshaft. Current maintenance involving disassembly of the cylinder-piston group is performed due to the wear of piston rings and cylindrical bushings.
\end{abstract}

\section{Introduction}

At present, open-pit mining of coal on different ores is the most promising way of resource sector development [1-4]. One of the limiting factors for open-pit mining development is the reliability of quarry equipment [5-7], especially dump trucks.

In real operating conditions of sliding bearings and especially diesel pistons, a semi-liquid friction regime is observed.

The oil layer is disturbed by a change in pressure and direction of movement. When the oil film ruptures, the mating friction pair is worn. An increase in the wear of rubbing parts is accompanied by a proportional increase in the friction forces. Given this, we can write:

$$
\Delta=K_{t p} \cdot \varpi, \mu \mathrm{m}
$$

where $\varpi$ - specific work of friction forces.

It is believed that the rubbing parts of the engine undergo a normal process of abrasive wear, and a change in the geometric shapes of the rubbing pairs does not affect the intensification of wear.

The concept outlined is fully consistent with the common energy wear criterion, which represents the ratio of the volume of abrasion products to the work of friction forces, and also with the law of wear;

$$
\Delta(l)=K_{t p} * S_{t p} * f(l)
$$

*Correspondent author: gdb@kuzstu.ru 
where $K_{t p}=f(l)$ - equation of specific pressure diagram,

$S_{t p}-$ friction part.

A quantitative measure of the wear of rubbing parts is the weight of the abrasive wear product or the linear value of the mechanical abrasion of the parts. An indirect measure of the amount of wear above was the specific work of the friction forces. Then the indirect measure of wear per unit of time $\delta$ will be the loss of mechanical energy per unit of time due to friction in the engine. In fact, expression (1.1) can be represented as:

$$
\delta=\frac{\Delta}{\tau}=\frac{K_{t p}}{m_{c} \cdot F_{n}} \cdot N_{t p}
$$

where $m_{c}-$ number of cylinders,

$F_{n}-$ piston area.

\section{Results}

With the maximum allowable wear in operation in the crankshaft journals and cylinder liners, the diesel engine life will be:

$$
M=\tau \frac{\Delta_{n p} * m_{c} * F_{n}}{K_{t p} \cdot N_{t p}}
$$

By replacing in (1.4) the known expression,

$$
N_{t p}=\frac{m_{c} \cdot v_{h}}{4500 \cdot i} \cdot n \cdot P_{t p}
$$

We will get

$$
M=150 \cdot \frac{\Delta_{n p}}{\mathrm{~K}_{\mathrm{tp}}} \cdot \frac{1}{C_{n} \cdot P_{t p}}
$$

where $i$ - tact ratio, $i=1,2$;

$C_{n}$ - average piston speed, $\mathrm{m} / \mathrm{s}$;

$P_{t p}$ - average conditional friction pressure.

The variable factor from specific parameters:

$$
K_{m}=\frac{i}{C_{n} \cdot C_{t p}}
$$

It can be taken as a comprehensive criterion for the durability of engines with a comparative assessment of their wear.

The power of mechanical friction losses can be represented as:

$$
N_{t p}=\frac{M \cdot \varpi}{75}=\frac{\pi}{75 \cdot 30} \cdot M_{c} \cdot n
$$

Where $M_{c}$ - engine torque, $\mathrm{N} \cdot m$;

$\varpi-$ angular speed of rotation of the diesel engine shaft, $\mathrm{rad} / \mathrm{s}$.

The moment of resistance of the engine shaft rotation to the approximation is expressed by a known empirical dependence:

$$
M_{c}=K_{c} \cdot \sqrt{n \cdot \eta}
$$


Where $\eta$ - absolute oil viscosity parameter, $N \cdot s / \mathrm{m}^{2}$;

$K_{c}$ - constant coefficient depending on engine design features,

$$
N_{t p}=\frac{K_{c} \cdot \pi}{75 \cdot 30} \cdot n^{1,5} \cdot \eta^{0,5}=K_{c} \cdot n^{1,5} \cdot \eta^{0,5}
$$

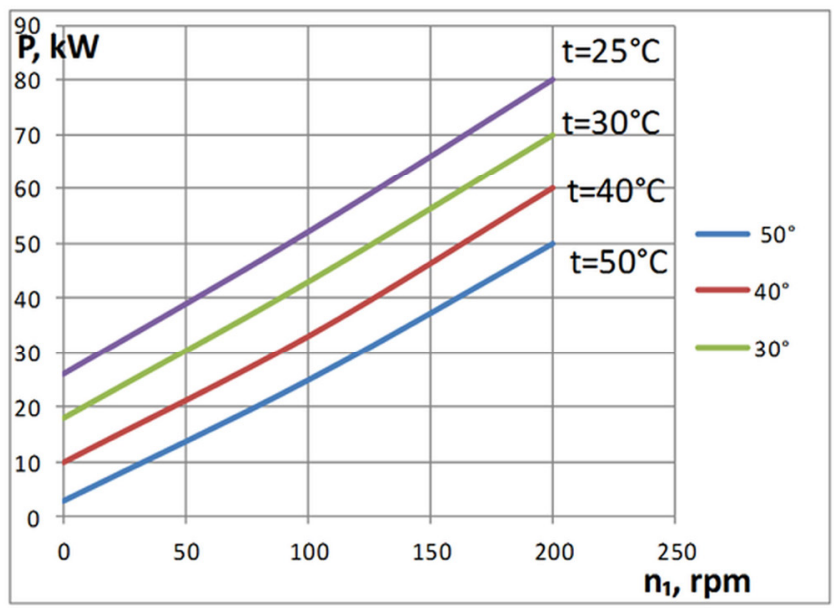

Fig. 1. Losses of mechanical energy due to friction depending on the speed of rotation of the D50M diesel engine shaft at various diesel oil temperatures.

The validity of the obtained dependence of the curves of loss of mechanical energy to friction depending on the temperature of diesel oil, the rotation speed of the D50M diesel shaft (Fig. 1).

Having substituted the expression (1.9) into (1.4), we obtain another function of the engine service life:

$$
M=\frac{\Delta_{n p^{*} m_{c} * F_{n}}}{K_{t p} \cdot K_{c}} \cdot \frac{l}{n^{1,5} \cdot \eta^{0,5}}
$$

In contrast to function (1.7), which represents the dependence of the engine life on the design parameters, this formula reflects the dependence of the durability of the diesel engine on operating conditions.

To determine the wear resistance of the most important working parts of a diesel engine, we replace the expression for the specific work of the friction forces in formula (1.1) by the superimposed energy method:

$$
\varpi_{t p}=\mu \cdot \rho \cdot v \cdot \tau
$$

Where $\mu$ - coefficient of sliding factor;

$\rho$ - normal specific pressure;

$v$ - relative sliding speed.

Thus,

$$
\mu=\tau_{\max }=\frac{\Delta_{t p}}{K_{t p} \cdot \mu \cdot \rho \cdot v}
$$

The product $(\rho \cdot v)$ is considered to be a measure of wear of rubbing parts. To ensure their durability, it should not exceed the norms developed by practice.

When determining the engine life by critical parts and assemblies which limit its service life and have legitimate wear, only the largest value of their local wear is of interest (Figure 1.2). The maximum local wear of the cylinder liner is in the area of the combustion chamber 
due to friction of the first piston rings, and for all crankshaft - it's in the inner generatrix of the journal (between the cheeks).

The work of friction forces is distributed in the diesel engine for friction: in cylinders (cylindrical bushings $\left.b_{c}=0.55-0.65\right)$, in the crankshaft journals $(b=0.35-0.45)$ and in the distribution mechanism in the pumps of all systems $\left(b_{n p}=0.03-0.05\right)$.

The working conditions of the friction forces in these zones should also be taken in practical calculations. In accordance with this, the most accurate value of the engine life can be obtained from the initial expression (1.13) taking on the local values of the coefficient of friction, sliding speed and normal pressures created by the pressure of the working gases on the piston and the inertia of the moving mechanism.

To establish the influence of the parameters of the working process on the wear of engine parts, it is necessary to carry out a number of transformations.
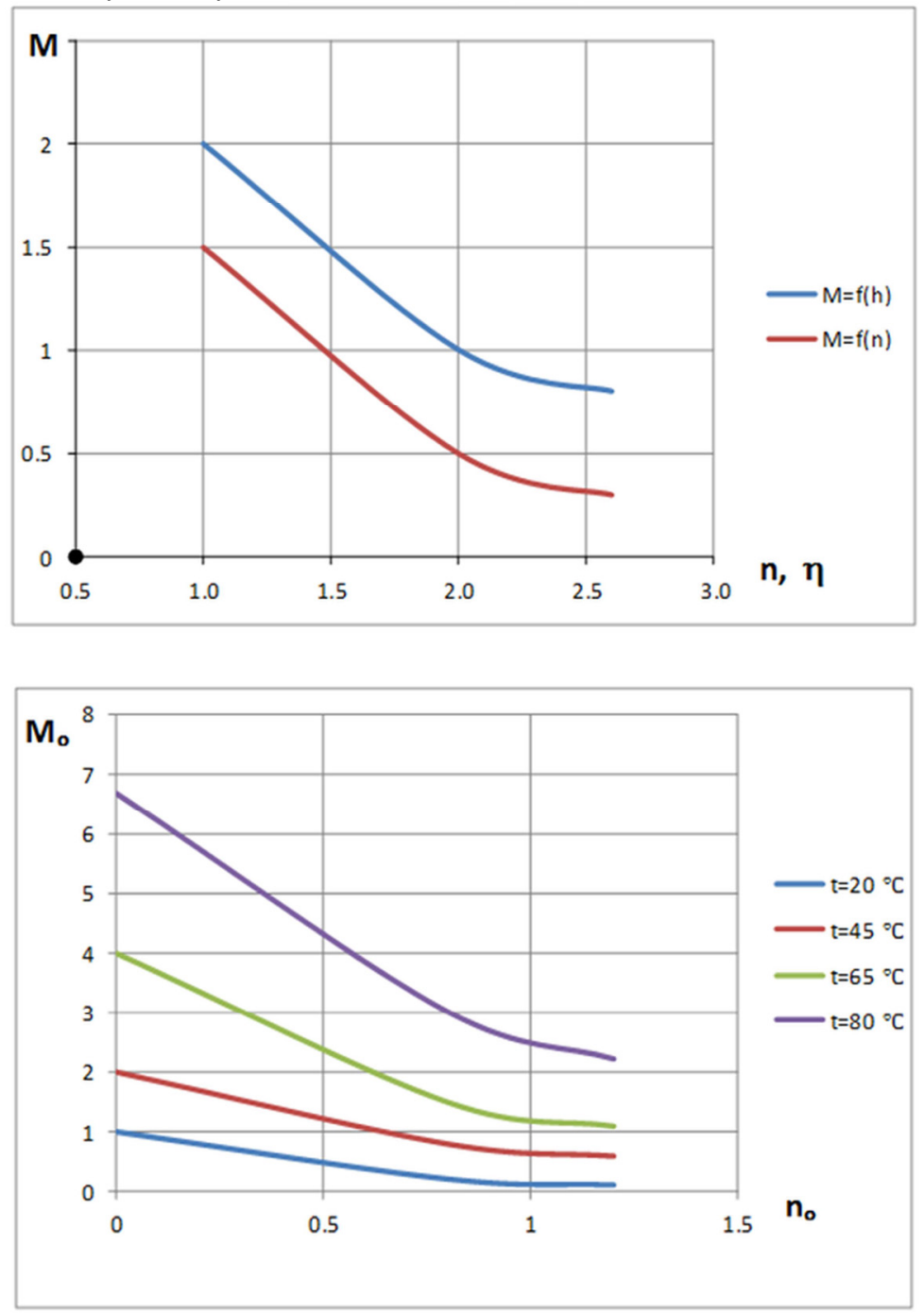

Fig. 2. Diesel life curves depending on shaft rotation speed (a) and absolute viscosity of diesel oil. 
The specific friction force $\mu \cdot$ Pas applied to the rubbing parts of an internal combustion engine is expressed in terms of a constant average friction pressure per cycle of the crankshaft rotation angle:

$$
\mu_{i} \cdot p_{i}=\beta_{i} \cdot \frac{P_{t p}}{i} \cdot \frac{F_{n}}{F_{t p_{i}}}
$$

where $F_{n}$ - piston area;

$F_{t p_{i}}$ - surface area considered in the diesel engine;

$\beta_{i}$ - fraction of the average friction pressure attributable to the considered friction surface.

The work of friction forces is distributed in the diesel engine into friction:

- in cylindrical bushings $\beta_{c}=0.55-0.65$

- in crankshaft journals $\beta_{n}=0.35-0.45$

- in the distribution mechanism and pumps of all systems $\beta_{n p}=0.03-0.05$ :

$$
F_{t p_{i}}=F_{n}=d_{n} \cdot l_{n}
$$

and its sliding peripheral speed is

$$
\vartheta_{n}=\frac{\pi \cdot d_{n} \cdot n}{60}=\frac{\pi \cdot d_{n} \cdot C_{i}}{2 \cdot S}
$$

Where $d_{n}$ and $l_{n}$ - journal diameter and length.

If we ignore the friction of the piston against the cylinder walls, the friction area is determined by the contact surface of the rings with the sleeve:

$$
F_{t p_{i}}=F_{c}=\pi \cdot D \cdot h_{k} \cdot m_{k}
$$

where $h_{k}$ - working width of the piston ring;

$m_{k}-$ number of rings on the piston.

Taking into account the above dependences, we obtain the following expression of the engine life by the wear of the main journals of the crankshaft:

$$
M=\frac{2 \cdot \lambda \cdot l_{n} \cdot \Delta_{n}}{\pi^{2} \cdot K_{t p} \cdot \beta_{n} \cdot D} \cdot \frac{i}{P_{t p} \cdot C_{c t}}
$$

and cylinder bushings

$$
M_{c}=\frac{m_{k} \cdot h_{k} \cdot \Delta_{n p}}{K_{t p} \cdot \beta_{n} \cdot D} \cdot \frac{i}{P_{t p} \cdot C_{c t}}
$$

where $D$ - cylinder diameter.

The resulting expressions are similar to each other and to formula (1.7). From formulas (1.10) and (1.11) it can be seen that the work of the friction forces and therefore the internal combustion engine service life are determined in operation by the speed and temperature conditions of its operation. In Figures 1.2a, 1.2b, diesel life curves are constructed in accordance with formula (1.11) depending on the shaft rotation speed and the absolute viscosity of diesel oil. The curves are given for the working range of change $n$ and $\eta$.

From the obtained expressions it can be seen that the engine life at variable operating conditions does not depend on the load. The mean friction pressure values included in the individual expressions are independent of the load and are determined by the same parameters as the moment of resistance:

$$
P_{t p}=f(n ; \eta)
$$

Where $n$ - number of crankshaft rotations, rpm. 
Hence, the idle and loaded modes at the same $h$ and $\eta$ have approximately the same effect on engine durability.

Thus, the diesel engine life does not depend on the following main factors: the degree of forcing, the diameter of the cylinder, crankshaft rpm, the rigidity of the structure and the stress level incorporated in it, as well as the specific pressures between the rubbing surfaces, which can indirectly be estimated by the specific weight of the engine. A significant impact on the engine life is exerted by the types of fuel and oil used, operating conditions, etc.

We determine now the dependence of the diesel engine life on the following parameters using the theory of multiple correlation: $P_{z} / P_{c}$ - an indicator of the degree of forcing and $g_{d i z}$ - specific weight of the diesel engine, $\mathrm{kgf} / \mathrm{hp}$.

The dependence of the diesel engine life on each of the arguments $D, n, P_{z} / P_{c}, g_{d i z}$ at the beginning was determined by the pair correlation method, and then the multiple correlation equation was deduced, taking into account the combined effect of the above parameters on the durability of the diesel engine.

We consider the linear correlation of motor life and cylinder diameter. The tightness indicators of the relationship between the two values is the correlation coefficient calculated by the formula:

$$
r_{M D}=\frac{\frac{\Sigma M \cdot D}{m}-\bar{M} \cdot \bar{D}}{\sigma_{M} \cdot \sigma_{D}}
$$

Where $r_{M D}$ - correlation coefficient;

$m$ - number of initial values in the statistical sampling (there were 17).

The average value of the engine service life and cylinder diameter

$$
\begin{aligned}
& \bar{M}=\frac{\Sigma M}{m} \\
& \bar{D}=\frac{\Sigma D}{m}
\end{aligned}
$$

The mean square deviation characterizing the degree of dispersion of the actual values of the motor life and cylinder diameter from the average value

$$
\begin{aligned}
& \sigma=\sqrt{\frac{\Sigma M^{2}}{m}-\bar{M}^{2}} \\
& \sigma=\sqrt{\frac{\Sigma D^{2}}{m}-\bar{D}^{2}}
\end{aligned}
$$

The correlation coefficient in the linear form of the relationship between $M$ and $D$ equals 0.972 , and the linear correlation equation has the following form

$$
M=(-7486.6)+1170.2 \cdot D
$$

i.e. the more $D$, the more $M$. The correlation coefficient between the parameters $M$ and $h$ is equal $(-0.88)$, and the correlation equation has the following form:

$$
M=41560-22 \cdot n
$$

The ratio $P_{z} / P_{c}$ is taken as an indicator of the degree of diesel forcing. The correlation coefficient for the dependence of motor life on the degree of forcing equals $(-0.633)$, and the dependence itself is described by the equation:

$$
M=39650-1974.8 \cdot P_{z} / P_{c}
$$


Calculation of the dependence of the engine service life on $P_{n} \frac{N_{c}}{\Sigma F} \cdot N_{c}$ shows that the correlation coefficient between $M$ and these values is much below 0.5 .

The specific weight $g_{d i z}$ of the diesel engine significantly affects the engine service life, which confirms the numerical value of the correlation coefficient, which is equal to 0.925 , the relationship between $M$ in $g_{d i z}$ characterizes the equation:

$$
M=11050+1250 \cdot g_{d i z}
$$

When studying the correlation dependence of the diesel engine service life on four factors, we limit ourselves to the simplest and most important for practical calculations case of the straight-line correlation described in general terms by the equation:

$$
M=\beta_{1}+\beta_{2} \cdot D+\beta_{3} \cdot n+\beta_{4} \cdot \frac{P_{z}}{P_{c}}+\beta_{5} \cdot g_{d i z}
$$

For the convenience of calculation, we express all the variables and the dependencies between them on a standardized scale and, after a series of mathematical transformations, we obtain a system of normal equations:

$$
\begin{aligned}
r_{M D}= & \beta_{2}+\beta_{3} \cdot r_{D n}+\beta_{4} \cdot r_{D K} n+\beta_{5} \cdot r_{D g_{d i z}} \\
r_{M n}= & \beta_{2} \cdot r_{D n}+\beta_{3}+\beta_{4} \cdot r_{n K}+\beta_{5} \cdot r_{n g_{d i z}} \\
r_{M K}= & \beta_{2} \cdot r_{D K}+\beta_{3} \cdot r_{n K}+\beta_{4}+\beta_{5} \cdot r_{K g_{d i z}} \\
& r_{M g_{d i z}}=\beta_{2} \cdot r_{D g_{d i z}}+\beta_{3} \cdot r_{n g_{d i z}}+\beta_{4} \cdot r_{K g_{d i z}}+\beta_{5}
\end{aligned}
$$

Where $r_{M D}, r_{M n}, r_{M K}, r_{M g_{d i z}}-$ correlation coefficients;

$\beta_{2}, \beta_{3}, \beta_{4}, \beta_{5}-$ standardized coefficients of the multiple regression equation;

$K-$ ratio $P_{z} / P_{c}$.

Having determined the correlation coefficients $r_{M D}, r_{D K}, r_{D g_{d i z}}, r_{n K}, r_{n g_{d i z}}, r_{K g_{d i z}}$ and using the previously obtained pair correlation coefficients, we find the numerical values of the standardized coefficients. Substituting the values of these coefficients into the standardized equation,

$$
\begin{gathered}
M-\bar{M}=\beta_{3} \cdot(D-\bar{D}) \cdot \frac{\sigma_{M}}{\sigma_{D}}+\beta_{2} \cdot(n-\bar{n}) \cdot \frac{\sigma_{M}}{\sigma_{n}}+\beta_{4} \cdot(K-\bar{K}) \cdot \frac{\sigma_{M}}{\sigma_{K}}+\beta_{5} \cdot\left(g_{d i z}-\overline{g_{d i z}}\right) \\
\cdot \frac{\sigma_{M}}{g_{d i z}}
\end{gathered}
$$

we obtain the multiple correlation equation characterizing the dependence of the diesel engine service life on $D, n, P_{z} / P_{c}, g_{\text {диз }}$

$$
M=5150+637 \cdot 6.8 \cdot D+279 \cdot P_{z} / P_{c}+529 \cdot g_{d i z}
$$

\section{Conclusion}

The formula (1.32) obtained for calculating the motor service life can be used to determine the durability of diesel engines.

The formula (1.32) is valid for the nominal mode of diesel operation. Knowing the average values during operation in certain climatic zones and at specific diesel operating modes, we can approximately assess the effect of operating modes on the engine service life. 
The results of the reliability analysis can be used in development of standards for maintenance and repair of open-pit dump trucks, in predicting the failure of units, assemblies and dump trucks in general.

\section{References}

1. T. Gvozdkova, M. Tyulenev, S. Zhironkin, V. A. Trifonov, Yu. M. Osipov, IOP Conf. Ser.: Earth Environ. Sci., 50:1, 012010 (2017)

2. G. Buyalich, K. Buyalich, M. Byakov, E3S Web Conf., 21, 03018 (2017)

3. D. Szurgacz, J. Brodny, Sustainability, 11:9, 2570 (2019). DOI: doi.org/10.3390/su11092570

4. O. Litvin, M. Tyulenev, S. Zhironkin, S. Prokopenko, Acta Montanistica Slovaca, 22:2, 146-152 (2017)

5. G.D. Buyalich, B.A. Aleksandrov, Y.A. Antonov, V.V. Voyevodin, Journal of Mining Science, 36:5, 487-492 (2000)

6. M. Tyulenev, O. Litvin, S. Zhironkin, M. Gasanov, Acta Montanistica Slovaca, 24:2, 88-97 (2019)

7. M. Tyulenev, O. Litvin, M. Cehlár, S. Zhironkin, M. Gasanov, Acta Montanistica Slovaca, 22:3, 296-302 (2017) 